\title{
Transient overshoot extensional rheology of long-chain branched polyethylenes: Experimental and numerical comparisons between filament stretching and cross-slot flow
}

Hoyle, D.M.; Huang, Qian; Auhl, D.; Hassell, D.; Rasmussen, Henrik K.; Skov, Anne Ladegaard; Harlen, O.G.; Hassager, O.; McLeish, T.C.B.

Published in:

Journal of Rheology

Link to article, DOI:

$10.1122 / 1.4767982$

Publication date:

2013

Document Version

Publisher's PDF, also known as Version of record

Link back to DTU Orbit

Citation $(A P A)$ :

Hoyle, D. M., Huang, Q., Auhl, D., Hassell, D., Rasmussen, H. K., Skov, A. L., Harlen, O. G., Hassager, O., \& McLeish, T. C. B. (2013). Transient overshoot extensional rheology of long-chain branched polyethylenes:

Experimental and numerical comparisons between filament stretching and cross-slot flow. Journal of Rheology, 57(1), 293-313. https://doi.org/10.1122/1.4767982

\section{General rights}

Copyright and moral rights for the publications made accessible in the public portal are retained by the authors and/or other copyright owners and it is a condition of accessing publications that users recognise and abide by the legal requirements associated with these rights.

- Users may download and print one copy of any publication from the public portal for the purpose of private study or research.

- You may not further distribute the material or use it for any profit-making activity or commercial gain

- You may freely distribute the URL identifying the publication in the public portal 


\section{Transient overshoot extensional rheology of long-chain branched polyethylenes: Experimental and numerical comparisons between filament stretching and cross-slot flow}

D. M. Hoyle, Q. Huang, D. Auhl, D. Hassell, H. K. Rasmussen et al.

Citation: J. Rheol. 57, 293 (2013); doi: 10.1122/1.4767982

View online: http://dx.doi.org/10.1122/1.4767982

View Table of Contents: http://www.journalofrheology.org/resource/1/JORHD2/v57/i1

Published by the The Society of Rheology

\section{Related Articles}

High shear rheometry using hydrodynamic lubrication flows

J. Rheol. 57, 197 (2013)

The effect of interfacial slip on the rheology of a dilute emulsion of drops for small capillary numbers

J. Rheol. 56, 1555 (2012)

Extensional rheometry at interfaces: Analysis of the Cambridge Interfacial Tensiometer

J. Rheol. 56, 1225 (2012)

A simple accurate method for measuring viscosity of liquid metals at high temperatures

J. Rheol. 56, 941 (2012)

Rheological modification of corn stover biomass at high solids concentrations

J. Rheol. 56, 649 (2012)

\section{Additional information on J. Rheol.}

Journal Homepage: http://www.journalofrheology.org/

Journal Information: http://www.journalofrheology.org/about

Top downloads: http://www.journalofrheology.org/most_downloaded

Information for Authors: http://www.journalofrheology.org/author_information

\section{ADVERTISEMENT}

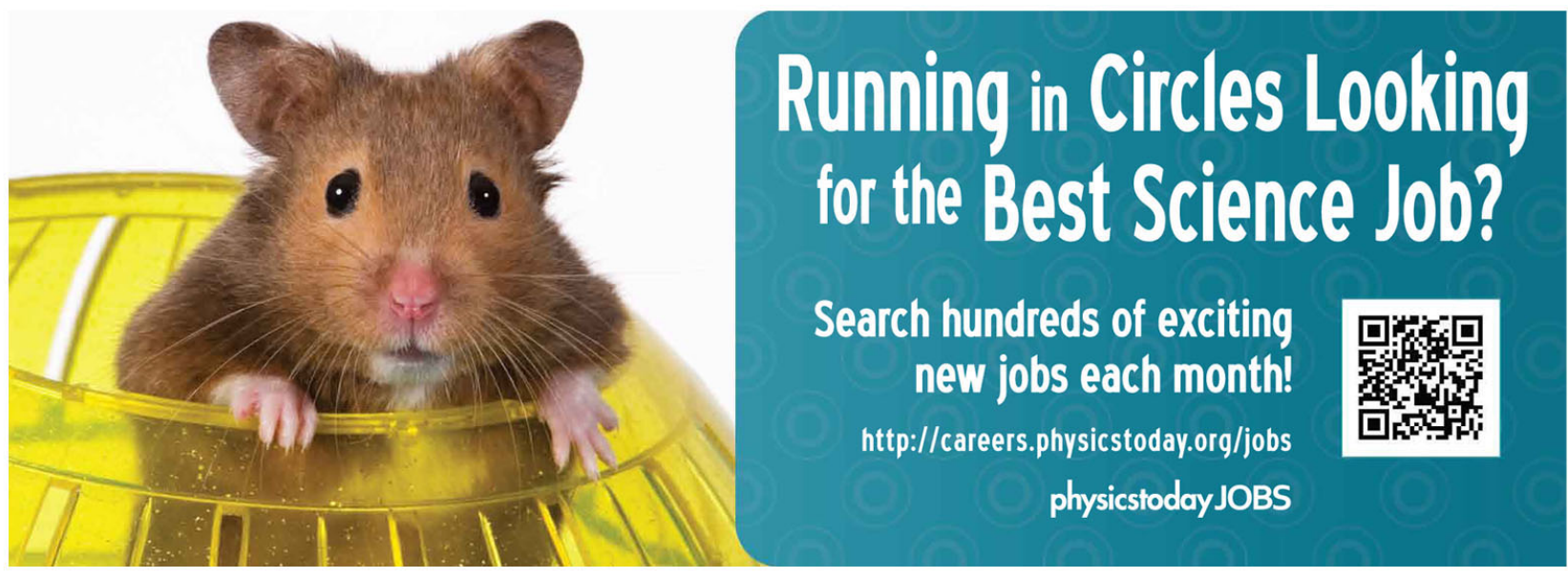




\title{
Transient overshoot extensional rheology of long-chain branched polyethylenes: Experimental and numerical comparisons between filament stretching and cross-slot flow
}

\author{
D. M. Hoyle ${ }^{\text {a) }}$ \\ Department of Physics, University of Durham, Durham DH1 3HP, \\ United Kingdom and Department of Applied Mathematics, University of Leeds, \\ Leeds LS2 9JT, United Kingdom \\ Q. Huang \\ Department of Chemical and Biochemical Engineering, The Danish Polymer \\ Centre, Technical University of Denmark, DK-2800 Kongens Lyngby, Denmark
}

$$
\text { D. Auhl }
$$

Interdisciplinary Research Centre for Polymer Science and Technology (IRC), University of Leeds, Leeds LS2 9JT, United Kingdom and Institute of Condensed Matter, Bio- and Soft Matter, Université Catholique de Louvain, B-1348 Louvain-la-Neuve, Belgium

\section{Hassell}

Department of Chemical Engineering, University of Cambridge, Cambridge CB2 3RA, United Kingdom and Institut Teknologi Brunei, Jalan Tungku Link, Gadong BE1410, Brunei Darussalam

H. K. Rasmussen

Department of Mechanical Engineering, The Danish Polymer Centre, Technical University of Denmark, DK-2800 Kongens Lyngby, Denmark

\section{A. L. Skov}

Department of Chemical and Biochemical Engineering, The Danish Polymer Centre, Technical University of Denmark, DK-2800 Kongens Lyngby, Denmark

$$
\text { O. G. Harlen }{ }^{\text {b) }}
$$

Department of Applied Mathematics, University of Leeds, Leeds LS2 9JT, United Kingdom

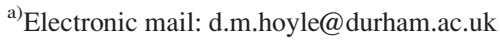

b) Author to whom correspondence should be addressed; electronic mail: o.g.harlen@maths.leeds.ac.uk
} 


\author{
O. Hassager \\ Department of Chemical and Biochemical Engineering, The Danish Polymer \\ Centre, Technical University of Denmark, DK-2800 Kongens Lyngby, Denmark
}

T. C. B. McLeish

Department of Chemistry, University of Durham, Durham DH1 3LE,
United Kingdom and Department of Physics, Durham University,
Durham DH1 3HP, United Kingdom

(Received 2 July 2012; final revision received 22 October 2012; published 4 December 2012)

\begin{abstract}
Synopsis
This work analyses the high-strain extensional behavior of long-chain branched polyethylenes, employing two novel extensional rheometer devices, the filament stretching rheometer and the crossslot extensional rheometer. The filament stretching rheometer uses an active feedback loop to control the imposed strain rate on a filament, allowing Hencky strains of around 7 to be reached. The cross-slot extensional rheometer uses optical birefringence patterns to determine the steady-state extensional viscosity from planar stagnation point flow. The two methods probe different strain-rate regimes and in this paper we demonstrate the agreement when the operating regimes overlap and explore the steadystate extensional viscosity in the full strain-rate regime that these two complimentary techniques offer. For long-chain branched materials, the cross-slot birefringence images show a double cusp pattern around the outflow centre line (named W-cusps). Using constitutive modeling of the observed transient overshoot in extension seen in the filament stretching rheometer and using finite element simulations we show that the overshoot explains the W-cusps seen in the cross-slot extensional rheometer, further confirming the agreement between the two experimental techniques. (C) 2013 The Society of Rheology. [http://dx.doi.org/10.1122/1.4767982]
\end{abstract}

\title{
I. INTRODUCTION
}

A long standing issue in characterizing polymer stress-strain relationships is their extensional response. It still remains a challenge both experimentally and constitutively to capture and explain the extensional stress response of polymer melts over a broad range of strain rates. The extensional stress growth coefficient provides an excellent material characterizing technique as the flow direction and gradient are parallel, and probes the chain stretching response. It is much more effective than shear flow in detecting differences in molecular structure [Bent et al. (2003)]. For branched polymer melts, the stress response usually exhibits some degree of strain hardening, where the tensile stress growth coefficient rises above the linear-viscoelastic reference case that is predicted using the Boltzmann superposition principle [Dealy (1990)].

Extensional flow is commonly achieved by uniaxial stretching [e.g., Cogswell (1972); Meissner (1971); Meissner and Hostettler (1994); Münstedt (1979); Sridhar et al. (1991)]. In a series of papers, Meissner, Münstedt, Laun, and co-workers investigated the strain-rate and stress dependence of the elongational viscosity as well as the recoverable strain in the steady state for low-density polyethylene (LDPE) melts [for example, Laun and Münstedt (1976, 1978); Raible et al. (1979)]. These constant strain-rate tests were performed on LDPE samples using a Meissner-type elongational rheometer with rotating clamps. These tests indicated that the tensile stress runs through a broad maximum as a function of strain. 
This effect was found to be even more pronounced at higher strain rates. However, Münstedt and Laun (1981) used the same Meissner-type apparatus to suggest that the stress maximum may be an artifact, because when the case of decreasing stress at high elongation occurs the sample homogeneity becomes insufficient to return significant data.

In subsequent studies, steady-state elongational viscosities were determined by creep tests in elongation [e.g., by Münstedt and Auhl (2005) for linear and long-chain branched (LCB) polypropylenes using a Münstedt type tensile rheometer]. In comparison to stretching experiments, a steady-state flow in elongation can already be reached in creep experiments for smaller strain rates. The steady-state values are independent of the flow type by which they are obtained and can be used to compare to the maximum values from constant strain-rate experiments. From the prescribed constant stress and the resulting steady-state elongational rate, the steady-state elongational viscosities were compared to the maximum viscosities from stressing experiments. The difference in the results when comparing steady-state extensional creep and constant strain-rate (maximums) measurements was similar for linear and weakly branched materials but somewhat larger for the materials with the highest long-chain branching.

Nonlinear elongational flow behavior is often explored using a double roller stretching device, the Sentmanat elongational rheometer \{SER, Xpansion Instruments [Sentmanat (2004)]\} attached to a standard shear rheometer. Typically different Hencky strain rates between 0.001 and $30 \mathrm{~s}^{-1}$ are applied to specimens with a width from 3 to $10 \mathrm{~mm}$ and a thickness of about $1 \mathrm{~mm}$ [cf., for example, Münstedt and Auhl (2005)]. However, the extensional data obtained from the SER never reach a steady-state plateau as stretching experiments are prone to sample inhomogeneity and sample rupture [cf. McKinley and Sridhar (2002); Minoshima and White (1986a, 1986b); Aho et al. (2010a, 2010b)] and these experiments are limited to Hencky strains less than 4.

A maximum in the transient extensional viscosity has been reported for LDPE [Meissner (1985)] but a steady-state stress after the maximum was not observed. More recently, Hassager and co-workers have used a filament stretching rheometer (FSR) with active feedback [Bach et al. (2003); Rasmussen et al. (2005)] to measure the elongational viscosity of LDPEs at strains beyond the onset of localized necking of the sample. However, even with active feedback a true steady-state flow condition is impossible to establish in filament stretching flows [see, for instance, McKinley and Sridhar (2002)] since an unlimited deformation is required and the sample cross-sections become very small. Despite this, the observation of an effective steady-state stress at high strains following a stress maximum was reported for two LDPE melts (Lupolen3020D and Lupolen1840D) [Rasmussen et al. (2005)]. In subsequent work, a similar high-strain "steady-state" stress plateau following a stress maximum has been reported for a model branched (Pompom) polystyrene of known architecture [Nielsen et al. (2006)].

Stretching devices have also been developed to measure the response of materials to planar extensional flow [Laun and Schuch (1989); Meissner et al. (1982); Meissner et al. (1981)]. In the linear-viscoelastic limit, the extensional viscosities for these two flow types differ by a factor of $4 / 3$. However, this difference decreases in the nonlinear strain hardened regime where the stress response tends to the same steady-state values [as seen, for example, in the LDPE measurements of Laun and Schuch (1989)] captured by the multimode Pompom model [Inkson et al. (1999)]. Consequently in this paper, we shall compare uniaxial and planar extensional data.

Auhl et al. (2011) used a closed cell stagnation point (SP) flow to measure the planar steady-state extensional response of various polyethylenes produced using flow induced birefringence (FIB) patterns. Much work has been done to investigate complex flow geometries [e.g., Boukellal et al. (2011); Coventry and Mackley (2008); Crowley et al. 
(1976); Frank and Mackley (1976); Janeschitz-Kriegl (1983); Macosko et al. (1980); Schoonen et al. (1998); Scrivener et al. (1979); Soulages et al. (2008); Verbeeten et al. (2001); Winter et al. (1979)], often comparing constitutive predictions to FIB images. Compared to filament stretching flows, the SP geometries have the advantage that flow properties can be investigated without free surface flow [Minoshima and White (1986b)]. However, the flow history at other points in flow is more complex and so only at the SP is the stress the result of simple planar elongational flow.

The use of FIB to measure stress anisotropy requires the stress-optical rule to remain linear in the strain-hardening regime. Simultaneous measurements of the tensile stress and birefringence as a function of time at constant tensile strain rate for LDPE were performed by Kotaka et al. (1997) and indicated that a linear stress-optical rule can be assumed up to about $1 \mathrm{MPa}$ in the case of polyethylene. Koyama and Ishizuka (1989) performed rheo-optical measurements in elongation on molten commercial LDPE using a Meissner-type elongational rheometer and found a linear stress-birefringence relationship in the birefringent patterns, even when strain hardening is seen in the nonlinear elongational viscosity. This is consistent with the relevant polymer physics: Strain-hardening onset corresponds to stretch at the level of entanglement strands, whereas a breakdown in the stress-optical linear rule corresponds to stretch at the level of segments [McLeish (2002)].

Constitutive modeling of polymer melts has evolved to focus on relating molecular architecture to macroscale stress response. For LCB polymer melts, various versions of the Pompom model by McLeish and Larson (1998) have been used [e.g., Clemeur et al. (2003); McLeish (2002); Öttinger (2001); Verbeeten et al. (2001)]. The molecular stretch function model [Wagner and Rolon-Garrido (2008)] predicts an overshoot in transient extension. In this paper, we use an adaptation of the Pompom model that incorporates a transient overshoot in extension. This is an empirical alteration that we have incorporated into our finite element simulations. The physical mechanism of the overshoot is yet to be fully resolved, but the Pompom model used here helps demonstrate the link between the transient extensional overshoot and the flow features seen in cross-slot flow experiments.

Various constitutive equations have been examined previously in a cross-slot geometry. For example, Bogaerds et al. (1999) showed that the Giesekus and Phan-Thien Tanner models fail to predict downstream principal stresses in cross-slot geometry for polymer solutions due to a failure of capturing extensional stresses. Abedijaberi et al. (2009) investigated the flow of LDPE branched polymer melts in a lubricated cross-slot channel by experiments and flow simulations. Hassell et al. (2009) compared the birefringence patterns of a range of moderately branched (metallocene-catalyzed) polyethylenes with the predictions of the Pompom model [Blackwell et al. (2000)]. As the degree of branching increases they observed a transition in the birefringence pattern from a single cusp along the outflow axis to a double cusp, which they named (W-cusps). This pattern indicates that the position of maximum stress difference is no longer at the SP, but away from the outflow axis. While the Pompom model was able to reproduce the general levels of principal stress difference (PSD), it did not reproduce the W-cusp pattern. Our subsequent extensive exploration of the constitutive parameter space of the multimode Pompom model, including fully three-dimensional simulations, has also failed to produce this phenomenon.

In this paper, we compare extensional stress measurements on a set of LCB polymers using two different extensional rheometers: The actively controlled FSR [Bach et al. (2003)] and the cross-slot extensional rheometer (CSER) [Auhl et al. (2011)]. We show that for range of strain-rates where both instruments can be used that the extensional stress at large strains is in good agreement, suggesting that it is possible to define an 
effective "steady-state" extensional viscosity for these materials. Finally, we show that by incorporating a transient maximum in the extensional stress response into the constitutive model we are able to reproduce the W-cusps.

\section{EXPERIMENTAL}

Three different branched polyethylene melts were studied: A highly branched LDPE Dow 150R and two moderately branched high-density polyethylenes (HDPE) HDB4 and HDB6 (see Table I). These materials have been used in a number of previous rheological studies. The linear rheology of HDB4 and HDB6 has been reported by Das et al. (2006) and that of Dow150R by Hassell et al. (2008). The shear and uniaxial extension rheology were measured at the same temperature as the subsequent cross-slot and FSR experiments [Auhl et al. (2011)]. Shear flow experiments were conducted with an ARES rheometer (Advanced Rheometric Expansion System, Rheometric Scientific) in order to obtain both the linear rheological and nonlinear shear flow behavior. The nonlinear flow behavior in uniaxial elongation was measured using the uniaxial stretching device SER (Xpansion Instruments) attached to the ARES rheometer [Sentmanat (2004)]. Specimen dimensions (compression molded to $1 \mathrm{~mm}$ thick and $10 \mathrm{~mm}$ wide samples) at test temperature were corrected to consider thermal expansion by using the room-temperature density and the thermal expansion coefficient of the samples. All of the rheological experiments were carried out under a nitrogen atmosphere. Further rheological tests to assess the thermal stability of the samples were conducted to ensure that the molar mass distribution and the molecular structure did not change during experiments. Thermal stability of at least $10^{4} \mathrm{~s}$ was found for all materials.

\section{A. Filament stretching rheometry}

Extensional measurements using the SER rheometer are limited to cases where the sample remains homogeneous. In order to explore higher strains as the deformation becomes inhomogeneous, we require an experiment in which the material whose stress is being measured experiences a kinematically steady extensional flow. These measurements are performed with an FSR equipped with an oven to allow measurements up to about $200^{\circ} \mathrm{C}$ [Bach et al. (2003)]. The key feature of this rheometer is that it uses active feedback through the measurement of the midplane diameter to control the strain rate at the midplane of the filament, which provided that a critical strain rate $\dot{\epsilon}_{s a g}=\rho g L_{0} / \eta_{0}$ is exceeded [McKinley and Sridhar (2002)] in the thinnest part of the filament. Therefore, on-line measurements of the midplane diameter serve the dual purpose of recording the actual strain and strain-rate at the midfilament plane and providing input for the feedback control on the plate motion to achieve desired kinematics. Specifically, the Hencky strain and the mean value of the stress difference over the midfilament plane [Szabo (1997)] are calculated from observations of the diameter $D(t)$ and the force on the bottom plate $F(t)$ as

TABLE I. Material properties of polyethylenes studied.

\begin{tabular}{lcccccr}
\hline \hline Sample & Code & $M_{W}(\mathrm{~kg} / \mathrm{mol})$ & $M_{W} / M_{N}$ & $\mathrm{~T}\left({ }^{\circ} \mathrm{C}\right)$ & $\eta_{0}(\mathrm{kPa} \mathrm{s})$ & $\bar{\tau}_{b}(\mathrm{~s})$ \\
\hline LDPE1 & Dow150R & 242 & 11 & 160 & 368 & 428 \\
HDPE1 & HDB4 & 96 & 2.1 & 155 & 200 & 56 \\
HDPE2 & HDB6 & 68 & 2.2 & 155 & 50 & 28 \\
\hline \hline
\end{tabular}




$$
\epsilon(t)=-2 \ln \left(D(t) / D_{0}\right)
$$

and

$$
\left\langle\sigma_{z z}-\sigma_{r r}\right\rangle=\frac{F(t)-m_{f} g / 2}{\pi R(t)^{2}},
$$

where the angular brackets denote an average over the symmetry plane, $R(t)=D(t) / 2$ is the radius of the filament, $g$ the gravitational acceleration, and $m_{f}$ the weight of the polymer filament. Consequently on the assumption that the stress is uniform across the midplane, the force measurement gives the normal stress difference at the midplane of the filament where the fluid has experienced a constant extension-rate, even though the overall extension-rate of the filament is nonuniform. Each material was tested a number of times to ensure reproducibility of the results.

Since the initial sample length is $L_{0}=2.5 \mathrm{~mm}$ compared with a plate radius $R_{0}=4.5 \mathrm{~mm}$, the initial sample aspect is small, and consequently at small strains not all of the stress difference is due to the extensional viscosity. Part of the stress difference comes from a radial pressure variation in the cross-section due to the shear flow that is unavoidable at small aspect ratios. To compensate for this effect, we define the corrected transient uniaxial elongation viscosity by

$$
\bar{\eta}_{\text {corr }}^{+}=\frac{\left\langle\sigma_{z z}-\sigma_{r r}\right\rangle}{\dot{\epsilon}_{0}}\left(1+\frac{\exp \left(-5 \epsilon / 3-\Lambda_{0}^{3}\right)}{3 \Lambda_{0}^{2}}\right)^{-1},
$$

where $\Lambda_{0}=L_{0} / R_{0}$ is the initial aspect ratio. The correction is a modification [Rasmussen et al. (2010)] of the relation derived from a lubrication analysis at small strains [Spiegelberg et al. (1996)]. For large strains, the correction vanishes and the radial variation of the stress in the symmetry plane becomes negligible [Kolte et al. (1997)].

\section{B. Cross-slot extensional rheology}

The cross-slot flow experiments to measure the steady planar extensional rheology were performed using the CSER, in which a cross-slot insert is used in the Cambridge multipass rheometer (MPR) [Mackley et al. (1995)]. This instrument allows simultaneous measurement of pressure and optical birefringence as detailed in Coventry and Mackley (2008) and used for a number of different polymer melt flow studies [e.g., Hassell and Mackley (2008, 2009); Hassell et al. (2009)]. The birefringence was measured using a circularly polarized monochromatic light beam of $514 \mathrm{~nm}$ using polarizers and quarter waveplates either side of the optical test section which contained stress free quartz windows. The stress-induced birefringence patterns were captured by a digital video camera [Collis and Mackley (2005)]. From the top and bottom reservoirs, the polymer material is driven in opposite directions along two perpendicular channels by pistons at a controlled rate through the cross-slot into two horizontal side channels capped by slave pistons [Fig. 1(a)]. Thereby, the material is maintained within the MPR and can be forced back by nitrogen pressure through the cross-slot insert into the top and bottom reservoirs for subsequent runs. The cross-slot geometry insert used in this study consists of four perpendicular, intersecting coplanar channels with a depth of $10 \mathrm{~mm}$ and aspect ratio of approximately 7 [Fig. 1(b)]. This generates a pure and controllable elongational deformation in the neighborhood of the stagnation line along the middle section of the centreaxis of the cross, but essentially simple shear near the outer walls, e.g., Coventry and Mackley (2008) and Hassell et al. (2008). Full three-dimensional flow simulations and experiments have been performed for both linear polystyrene [Lord et al. (2010)] and LCB-polyethylenes 

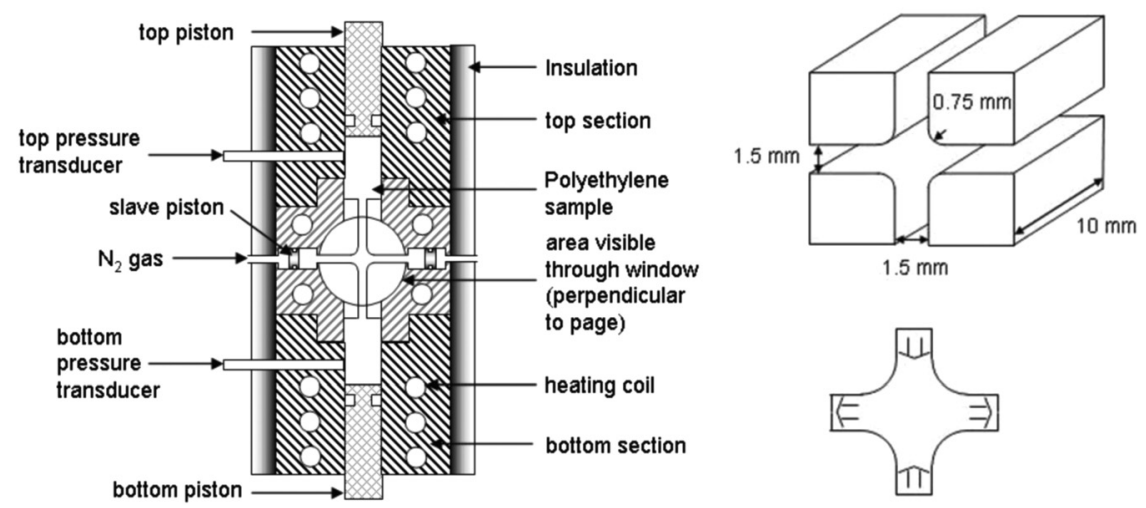

FIG. 1. (a) Schematic outlining the Cambridge MPR core and (b) the dimensions and flow direction for the crossslot geometry insert as used in the midsection of the MPR. The associated flow directions are indicated by arrows.

[Hoyle (2011)] in this geometry, where it was demonstrated that this aspect ratio is sufficiently large for the flow to be approximated as a two-dimensional planar flow within the experimental uncertainty of the stress measurements themselves, confirming previous simulation studies of Clemeur et al. (2004).

At steady state, a molecule at a point along the stagnation line experiences a constant extension-rate $\dot{\varepsilon}_{C}$ that is approximately proportional to the piston speed, but varies with polymer rheology due to changes in the flow pattern. To determine the extension-rate for each experiment, we performed flow simulations using a multimode Pompom model fitted to the measured rheology of the material. We have shown previously [Hassell et al. (2009); Hoyle (2011)] that changes in the velocity field at points around the stagnation line measured using laser Doppler velocimetry are captured by the Pompom model and further are dependent upon the level of LCB present in a material.

The steady-state elongational viscosity $\eta_{P}^{+}$is calculated from the tensile stress difference $\sigma_{s t d}$ along the stagnation line and the extension-rate $\dot{\varepsilon}_{C}$ there

$$
\eta_{P}^{+}=\frac{\sigma_{s t d}}{\dot{\varepsilon}_{C}} .
$$

Here, $\sigma_{s t d}=\left(\sigma_{x x}-\sigma_{y y}\right)$ is the PSD between the extensional $x$ and the compressional $y$ axes. This was determined from the fringe-counting [using the method detailed in Auhl et al. (2011)] as $\sigma_{s t d}=\frac{\Delta n}{C}$, where $C$ is the stress-optical coefficient. Stress-optical coefficients taken from Hassell et al. (2008) were used, which are in quantitative agreement with the range given in the literature for polyethylene of $1.2-2.4 \times 10^{-9} \mathrm{~Pa}^{-1}$ [Macosko (1994)]. According to the theory of rubber elasticity and experiments, the stress-optical coefficient is only weakly dependent on temperature [Koyama and Ishizuka (1989)] and so the same stress-optical coefficient was used for the experiments at 155 and $160^{\circ} \mathrm{C}$. In all cases, the stresses are below the $1 \mathrm{MPa}$ limit where the stress-optical rule is expected to be valid [Kotaka et al. (1997); Koyama and Ishizuka (1989); McLeish (2002)].

\section{CONSTITUTIVE MODELING AND FINITE ELEMENT SIMULATION TECHNIQUES}

The rheology for highly entangled polymers is strongly dependent on molecular topology. McLeish and Larson [McLeish and Larson (1998); McLeish (2002)] developed a 
constitutive model for branched polymers based upon the molecular theory for a melt of Pompom molecules, consisting of a backbone chain connecting two identical star polymers. Although derived with this particular molecular architecture in mind, the Pompom model captures the essential physics of branched melt rheology, and can be applied to more general architectures. A multimode Pompom model was introduced by Inkson et al . (1999) and later modified by Blackwell et al. (2000) to account for polydispersity and high complexities of multilevel branching.

In the multimode Pompom model, the extra stress tensor is formed as sum of products of the square of the backbone stretch, $\lambda_{i}(t)$, and orientation tensor, $S_{i}(t)$, from each mode,

$$
\boldsymbol{\sigma}=M^{-1} \sum_{i}^{N} G_{i} \lambda_{i}^{2}(t) \boldsymbol{S}_{i}(t),
$$

where $G_{i}$ are the linear viscoelastic moduli and $M^{-1}$ is a dimensionless constant that depends upon the equation used to obtain the orientation tensor. The original Pompom model uses an integral equation for which $M^{-1}=\frac{15}{4}$. However, since this form is computationally expensive a differential approximation based on the upper-convected Maxwell model was introduced for which $M^{-1}=3$. The differential model is more commonly used particularly in complex flow calculations as it is computationally simpler.

The differential approximation uses an auxiliary tensor $\boldsymbol{A}_{i}$, for each mode $i$, that satisfies the upper-convected Maxwell constitutive equation

$$
\frac{D A}{D t}=\boldsymbol{K} \cdot \boldsymbol{A}+\boldsymbol{A} \cdot \boldsymbol{K}^{T}-\frac{1}{\tau_{b}}(\boldsymbol{A}-\boldsymbol{I}) .
$$

Here (and from now on) we have suppressed the mode index, $i$ from $\boldsymbol{A}$ and $\tau_{b}$. The orientation is given by the unit tensor

$$
S=\frac{A}{\operatorname{tr} A}
$$

The dynamic equation describing the stretched backbone is given in Blackwell et al. (2000) and McLeish (2002) as

$$
\frac{D}{D t} \lambda(t)=\lambda(t) \boldsymbol{K}: S-\frac{1}{\tau_{s}}(\lambda(t)-1) e^{\nu *(\lambda(t)-1)},
$$

with $\nu^{*}=\frac{2}{q-1}$, for $\lambda$ up to a maximum value of $q$, at which point the branch points retract into the backbone tube.

A number of variants of this model have been developed including: a thermodynamically motivated differential model suggested by Öttinger (2001), the extended Pompom (XPP) differential model [Verbeeten et al. (2001)], and the double convected Pompom (DCPP) [Clemeur et al. (2003)]. These models drop the maximum stretch condition and improve the quantitative agreement with the integral model, in particular, to give a nonzero second normal stress difference in shear.

The various versions of the Pompom model have been shown to be able to quantitatively fit the transient shear and extensional behavior of a variety of different branched polymers up to the maximum in the extensional viscosity [Blackwell et al. (2000); Inkson et al. (1999); Verbeeten et al. (2001)]. However, the Pompom constitutive model cannot 
produce a transient overshoot in extension, such as that seen by Bach et al. (2003), Nielsen et al. (2006), and Rasmussen et al. (2005). This is because once the tube segments align with the flow axis, $\boldsymbol{K}: S \simeq \dot{\varepsilon}$ and the stretch equation reduce to an autonomous first order ordinary differential equation, and so $\lambda$ cannot overshoot its steady-state value.

The molecular origin of a transient overshoot in extensional flow is not currently understood. By incorporating the effect of tube pressure into the integral molecular stretch function, constitutive models [Rolon-Garrido and Wagner (2009); Wagner and Rolon-Garrido (2008)] were able to fit the experimental data on a melt of Pompom shaped molecules in Nielsen et al. (2006). However, the existence of a tube pressure that occurs when the tubes are deformed is a controversial idea whose molecular origin is unclear. The model predicts an overshoot followed by a steady state for all strain rates. The Pompom melt experimental data used did not reach steady state and so it could not be determined if the correct steady state was predicted by the model. In order to explore the consequences of extensional overshoots in the cross-slot flow, we choose at this point to proceed phenomenologically, suspending judgment on the underlying molecular reasons for such behavior.

To produce an overshoot, the Pompom model requires an additional stretch relaxation process that is dependent upon $S$. This is included as an additional relaxation rate $\tau_{*}^{-1}$ in the stretch equation (3.4) to give

$$
\frac{D \lambda}{D t}=\lambda \boldsymbol{S}: \boldsymbol{K}-\left(\frac{1}{\tau_{s}}+\frac{1}{\tau_{*}}\right)(\lambda-1) e^{\nu^{*}(\lambda-1)} .
$$

If this additional relaxation mechanism arises from advection by the flow, then it should be proportional to the extension-rate along the tube segments; hence, the relaxation time should be of the form

$$
\frac{1}{\tau_{*}}=f(\boldsymbol{S})|\boldsymbol{S}: \boldsymbol{K}|
$$

where $f$ is a function of the degree of alignment. Since this relaxation process becomes significant only once the tube segments become highly aligned, we choose a form for $f$ so that it switches from 0 to a positive value $C_{R}$ as the orientation tends to perfect alignment, by setting

$$
f(\boldsymbol{S})=C_{R}\left(\boldsymbol{S}: \boldsymbol{S}^{T}\right)^{\alpha}
$$

where the parameter $\alpha$ is used to define how aligned the material should be to trigger this additional relaxation. In a strong planar extensional flow (i.e., one for which the orientation Weissenberg number $\left.W i_{b}=\dot{\varepsilon} \tau_{b} \gg 1\right)$, the scalar $S: S^{T} \simeq 1-2 \exp (-2 \varepsilon)$ where $\varepsilon$ is the Hencky strain, $\dot{\varepsilon} t$ so that this function switches on the additional relaxation after a Hencky strain of approximately $\frac{1}{2} \log _{e}(2 \alpha)$. So that, for this relaxation to trigger after a Hencky strain of 2.6, requires a value of $\alpha$ of around 100. In uniaxial extension, $S: S^{T} \simeq 1-\frac{4}{W i_{b}} \exp (-2 \varepsilon)$.

The effect of varying the value of $\alpha$ is illustrated in Fig. 2. Figure 2(a) shows the extensional viscosity for a one mode Pompom model with $G=100 \mathrm{~Pa}, \tau_{b}=5 \mathrm{~s}, q=10$, $\tau_{s}=1.25 \mathrm{~s}$ for strain rates of $0.01,0.1,1$, and $10 \mathrm{~s}^{-1}$ (giving orientation Weissenberg numbers of $W i_{b}=\dot{\varepsilon} \tau_{b}=0.05,0.5,5$, and 50 and stretch Weissenberg numbers of $W i_{s}=\dot{\varepsilon} \tau_{s}=0.0125,0.125,1.25$, and 12.5 , respectively). The different lines show various choices of the power $\alpha$ with a fixed value of $C_{R}=2$. The figure shows that the steady-state value of the overshoot Pompom (OPP) model does not depend on $\alpha$, but that the value of $\alpha$ controls the strain at which the additional relaxation is triggered. 
(a)

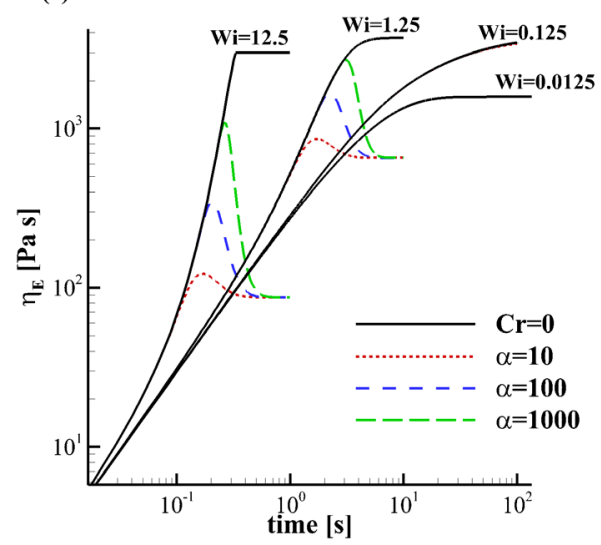

(b)

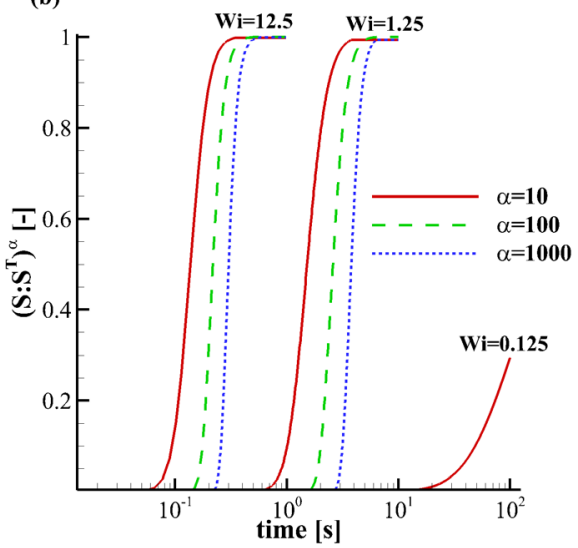

FIG. 2. (a) The transient extensional viscosity for a single mode overshoot Pompom model $\left\{G=100 \mathrm{~Pa}, \tau_{b}=\right.$ $\left.5 \mathrm{~s}, q=10, \tau_{s}=1.25 \mathrm{~s}\right\}$ in uniaxial extension. The four set of curves shows the effect of variations in the power law $\alpha$ from 10 to 1000 with $C_{R}=2$ for various stretch Weissenberg numbers $\left(W i_{s}=\dot{\varepsilon} \tau_{s}\right)$. As $\alpha$ is increased so does the amount of alignment needed for the extra relaxation time, $\tau_{*}$, to become dominant. This has the effect of delaying relaxation until a higher Hencky strain has been reached causing a bigger difference between the maximum and the steady-state extensional viscosity. (b) A plot of the measured alignment, $\left(S: S^{T}\right)^{\alpha}$, for the same stretch Weissenberg numbers. As $\alpha$ is increased so does the strain taken for the measured alignment to approach unity and thus delays the transient overshoot.

Figure 2(a) shows the value of $\left(S: S^{T}\right)^{\alpha}$ for the same one mode Pompom model in uniaxial extension, seen in Fig. 2(a). Only the highest strain rates (1 and 10 reciprocal seconds, or $W i_{s}=1.25$ and 12.5) align enough material for $\tau_{*}$ to become nontrivial, which coincide with the stretch Weissenberg number being greater than unity. For these two strain rates the various choices of $\alpha$ are shown and as before, the higher the value of $\alpha$ the larger the strain required to orientate the material sufficiently for this extra relaxation to contribute. For $\alpha=10,100$, and 1000, the Hencky strain at which steady state is observed is approximately 3,4 , and 5 , respectively.

Figure 3(a) shows the effect of varying $C_{R}$ for a fixed value of $\alpha=100$. As the parameter $C_{R}$ is increased so does the contribution of $\tau_{*}$ and the steady-state value of extensional viscosity decreases. Varying $C_{R}$ [cf. Fig. 3(a)] makes little difference in the Hencky strain at which steady state is achieved (approximately a Hencky strain of 4.5).

The inclusion of this additional stretch relaxation means that the backbone tubes are no longer fully extended at steady state. Indeed in Fig. 2(a), the maximum stretch condition $(q=10)$ was only reached for $C_{R}=0$. For lower values of $q$, it is still possible to achieve maximum stretch during the transient. This is illustrated in for the case $q=3$ in Fig. 3(b) where we consider the one mode Pompom model with $G=100 \mathrm{~Pa}, \tau_{b}=$ $10 \mathrm{~s}, \tau_{s}=2.5 \mathrm{~s}$ for choices of $q=3,10$, and 20 where the overshoot parameters are $\alpha=$ 1000 and $C_{R}=2$. Thus with the exception of cases where $q$ is small, the restriction of Eq. (3.5) to $\lambda<q$ becomes redundant and so removes one of the differences between the Pompom model and the XPP and DCPP models. However, the steady-state value still depends upon the value of $q$ since $\nu^{*}=\frac{2}{q-1}$.

In transient shear flow, the extra stretch relaxation is negligible (as the orientation tensor $\underline{\underline{S}}$ does not become sufficiently aligned) and the stress response is left unchanged.

\section{A. Finite element solutions}

Two-dimensional calculations of the flow of the Pompom model in the cross-slot geometry were performed using a finite element method to compute strain-rate and PSD. 
(a)

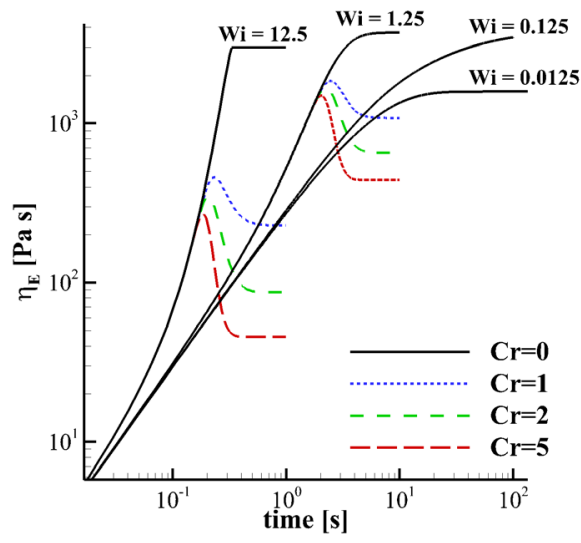

(b)

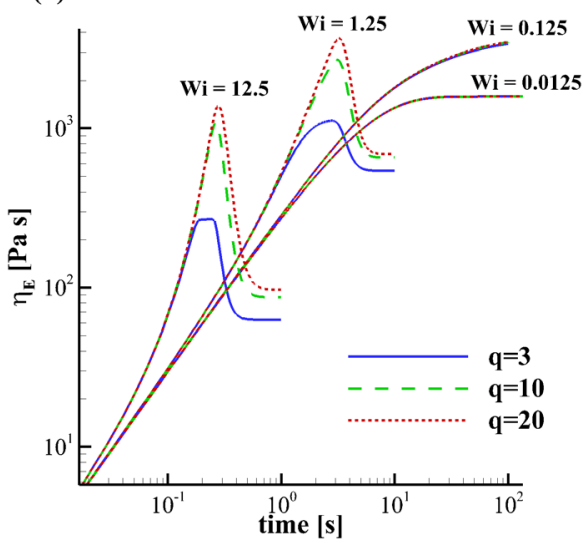

FIG. 3. (a) The transient extensional viscosity for a single mode overshoot Pompom model $\left\{G=100 \mathrm{~Pa}, \tau_{b}=5 \mathrm{~s}\right.$, $\left.q=10, \tau_{s}=1.25 \mathrm{~s}\right\}$ in uniaxial extension. The four set of curves shows the effect of variations in parameter $C_{R}$ from 1 to 5 with $\alpha=100$ for various stretch Weissenberg numbers $\left(W i_{s}=\dot{\varepsilon} \tau_{s}\right)$. The parameter $C_{R}$ does not affect the strain needed to achieve an overshoot but it does affect the magnitude of $\tau_{*}$ and thus determines the steady-state extensional viscosity. (b) The effect of the branch number $q$ on transient extensional viscosity for a single mode overshoot Pompom model $\left\{G=100 \mathrm{~Pa}, \tau_{b}=5 \mathrm{~s}, \tau_{s}=1.25 \mathrm{~s}, C_{R}=2, \alpha=100\right\}$ in uniaxial extension. The four set of curves shows the effect of variations in parameter $q$ from 3 to 20 for the same stretch Weissenberg numbers. In the case $q=3$ maximum stretch is achieved during the transient, but for larger values $q=10$ and $20, \lambda$ remains strictly less than $q$ for all time and the differences are due only to the change to $\nu^{*}$.

Details of the numerical scheme are given in the references [Tenchev et al. (2008); Lord et al. (2010)]. Due to symmetry, only one quarter of the cross-slot domain was calculated. Upstream we impose fully developed channel flow equivalent to the chosen experimental volume flux. Spatial convergence was checked by comparing solutions obtained on two different meshes of 970 and 2600 nodes. Simulations took up to $48 \mathrm{~h}$ on a $1.7 \mathrm{GHz}$ processor depending on mesh refinement and time step interval.

Since Eq. (3.2) allows unlimited stretch of the auxiliary tensor $\boldsymbol{A}$, a finitely extensible nonlinear elastic modification was made to the relaxation term to limit the trace of $\boldsymbol{A}$ to 1000. A penalty scheme was used to limit the stretch variable to the range $0 \leq \lambda(t) \leq q$, though as noted above this now occurs rarely due to the additional relaxation term.

\section{B. The OPP model in cross-slot flow}

In cross-slot flow, the difference between the maximum extensional stress and the steady-state value determines the size of the W-cusp and transient development of the extensional stress overshoot will determine the shape of the W-cusps. This is illustrated in Fig. 4 where in Fig. 4(a) the effect of varying the power law parameter on the shape of a PSD contour (of value $3 \mathrm{kPa}$ ) for a fixed $C_{R}=2$ is shown. At the lowest power law, $\alpha=10$ [see Fig. 2], the stretch relaxes at a lower strain and so the double cusps are shorter and wider than for the higher power laws.

Figure 4(b) shows how varying $C_{R}$ affects the position of the $0.3 \mathrm{kPa}$ contour. The $\mathrm{W}$-cusps are now all the same width (which as we have seen is associated with the value of $\alpha$ ); however, the length of the cusp is shorter for higher values of $C_{R}$ where the stretch relaxation is faster at high strains.

Figure 5 compares the magnitude of the PSD and the degree of orientation $S: S^{T}$ along the SP streamline as functions of the distance from the SP. Figure 5(a) shows the effect of changes to $\alpha$, which occurs mainly upstream from the SP. Here, the stress initially grows toward the SP and overshoots as a function of strain history, with the various power laws showing the same behavior as in Fig. 2. The small differences at and 
(a)

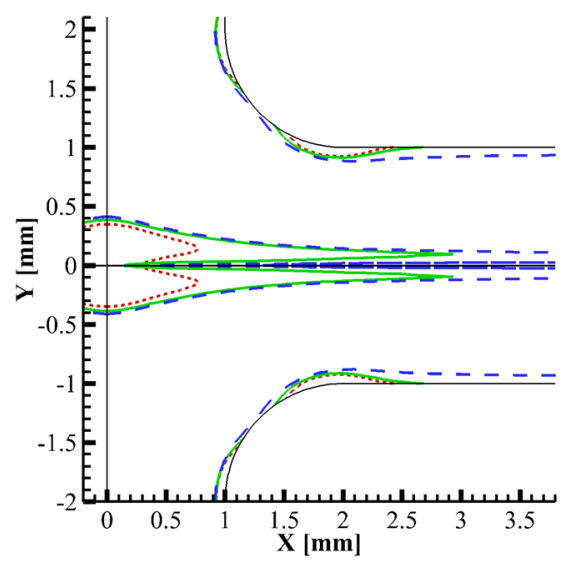

(b)

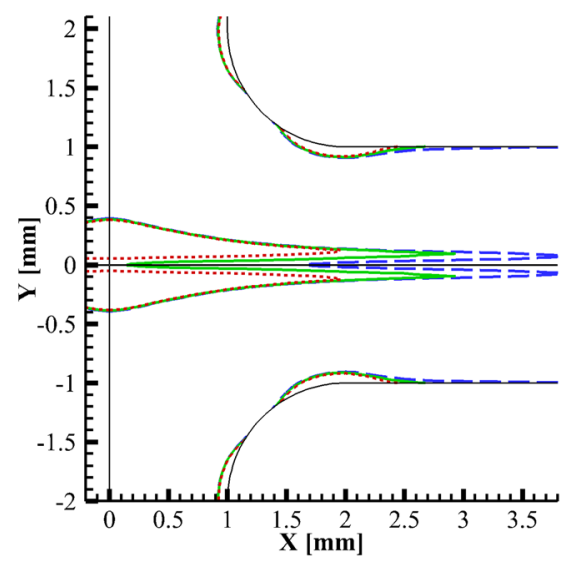

FIG. 4. (a) Computed contours of constant principal stress difference for the one mode Pompom model used in Fig. 2, examining how the power law $\alpha$ affects W-cusps in cross-slot flow. The dashed line shows $\alpha=10$, the solid line $\alpha=100$, and the dotted line $\alpha=1000$. (b) The effect of varying $C_{R}$ on the W-cusps in the cross-slot flow using the single mode Pompom models used in Fig. 3. The dotted line shows $C_{R}=5$, the solid line shows $C_{R}=2$, and the dashed line shows $C_{R}=1$.

downstream of the SP arise from flow modification, since $\alpha$ does not affect the steadystate stress. Similarly, the changes in the transient development of $S: S^{T}$ arise from changes in the flow pattern near the SP.

In Fig. 5(b), the three choices of $C_{R}$ do not change the values of $S: S^{T}$ demonstrating that it is $\alpha$ rather than $C_{R}$ which has the dominant effect on flow pattern. Similarly changes to the PSD occur only at high strains affecting the maximum and steady-state values of the PSD in the same way as in the uniaxial stretching flow shown in Fig. 3. The smallest maximum and steady state are associated with the largest value of $C_{R}$ since this provides the strongest stretch relaxation.

\section{RESULTS}

First, we compare the extensional viscosity measurements from the FSR, the Sentmanat extensional rheometer (SER), and the CSER. Figure 6 shows the steady-state
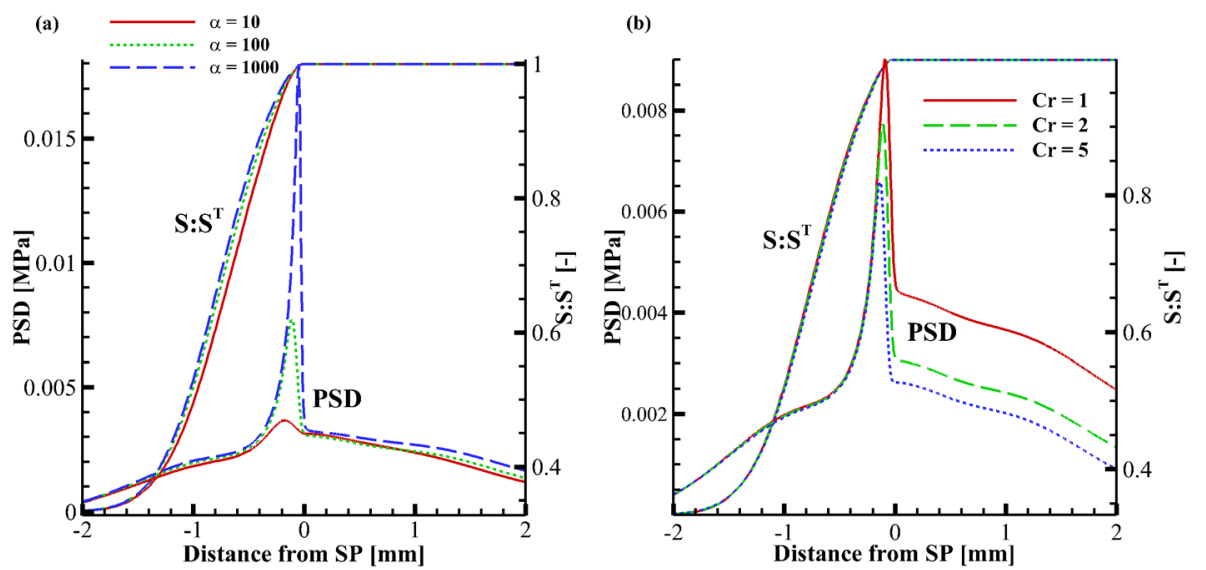

FIG. 5. PSD and $S: S^{T}$ along the SP streamline as a function of distance from the SP showing variations in (a) $\alpha$ and (b) $C_{R}$. Negative distance from the SP corresponds to the inlet channel and positive distance the outlet channel. 


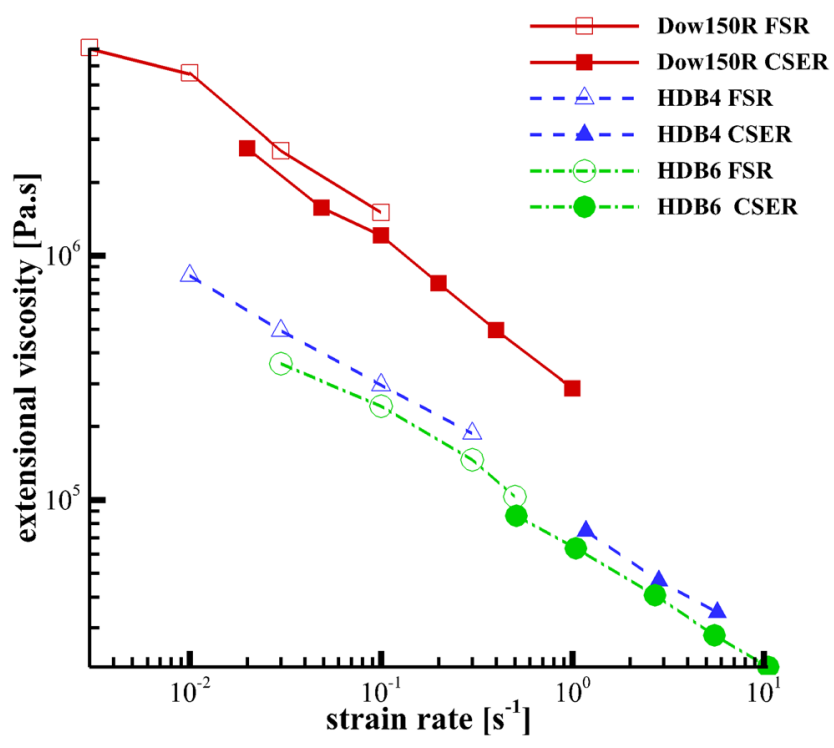

FIG. 6. A comparison of the steady-state extensional viscosity measurements from the FSR and the CSER for three polyethylene samples detailed in Table I. The open symbols show the FSR results and the closed symbols show the CSER data.

extensional viscosity measurements from the FSR and the CSER for the three polyethylene samples used in this work (cf. Table I). The FSR values are obtained from the average stress measurement at large strains and the CSER from the steady-state birefringence patterns. For two of the materials, there are sufficient data to examine the overlap of the two experimental methods (Dow150R and HDB6) where there is good agreement between the two. This is despite the differences in the nature of the flows, with the FSR being uniaxial and the CSER planar extensional flow. Even for HDB4, where there is a gap in the data, the steady-state stress values still show the close agreement to the same trend.

For strain rates where data are available, we also compare the transient stress growth in Figs. 7 and 8. Figure 7 shows the two LCB-HDPEs named HDB4 and HDB6 with data measured from the SER and CSER and Fig. 8 shows the LDPE Dow150R. Details of the materials are given in Table I. The comparison of transient data shows good agreement between the two filament stretching methods up to the point at which the SER samples rupture and break, at Hencky strains of around 4 (the FSR is capable of Hencky strains up to 7). As a general observation for these three materials, the transient build up of stress measured by the FSR is faster than that of the SER. Also, as anticipated the SER never goes far enough in Hencky strain to observe an extensional stress maximum or steady-state value. In contrast, the FSR data show a clear overshoot in stress. The presence of a steady state in Figs. 7 and 8 is obscured by the logarithmic axes that compress the large time results.

In Fig. 8, we also show the fit of the OPP model to both the transient startup flow (a) and for the steady-state extensional viscosity (b). The OPP model was parameterized with 12 modes. The linear Maxwell parameters $\left(\tau_{b i}\right.$ and $\left.G_{i}\right)$ were fitted to linear oscillatory rheology using RepTate. ${ }^{1}$ The nonlinear parameters $\left(\tau_{s i}\right.$ and $q_{i}$ for each mode, together with $C_{R}$ and $\alpha$ ) were fitted by hand to extensional data starting from values from

\footnotetext{
${ }^{1}$ Ramirez, J., and A. E. Likhtman, RepTate: Rheology of Entangled Polymers, Toolkit for Analysis of Theory and Experiment (2007), http://www.reptate.com
} 

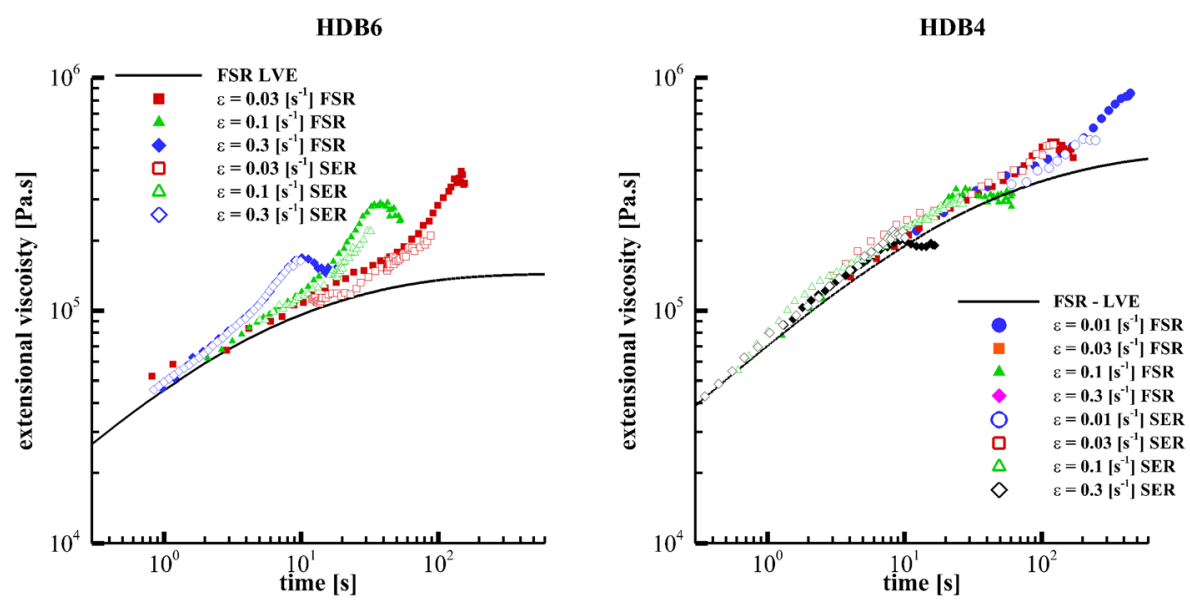

FIG. 7. A comparison between the transient extensional stress response as measured by the FSR (closed) and the SER (open) for HDB6 (left) and HDB4 (right). The figure shows a good agreement of the initial stress growth, until sample rupture limits the SER to Hencky strains of around 4.

a previous fit to the non-OPP model [Auhl et al. (2011); Hoyle (2011)]. The overshoot parameters are chosen to be the same for all modes to reduce the number of variables. This provides some unwanted overshoots at low strain rates, but we tolerate this as we are focusing on modeling the CSER birefringence images and so we aim to optimize the fit to this range of extensional strain rates.

The OPP model captures the startup extensional stress of Dow150R well [Fig. 8(a)] and for the three higher strain rates, where an overshoot in the stress is observed, the model also captures this. For the two lower strain rates, no large experimental overshoot is observed; however, the parameterized OPP model still displays one. This is reflected in the steady-state predictions of the FSR, the CSER, and the OPP model [Fig. 8(b)]. The OPP model fits the CSER data well but for lower strain rates the model under predicts the
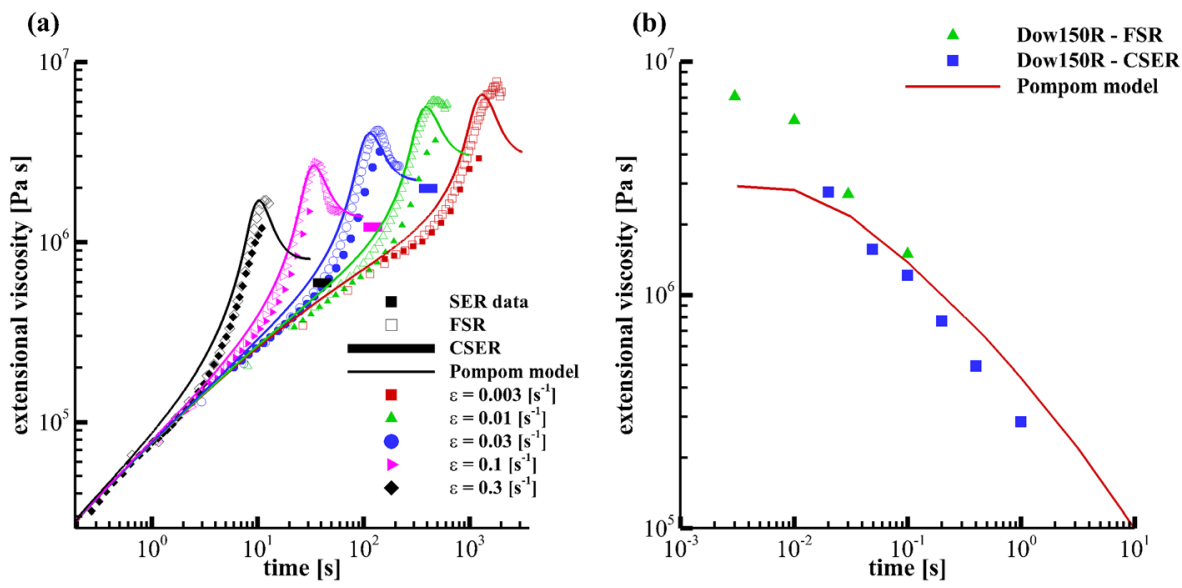

FIG. 8. (a) A plot comparing extensional data and OPP theory for Dow 150 R. Strain rates range from 0.003 to $0.3 \mathrm{~s}^{-1}$. The closed symbols show the SER data and the open symbols data from the FSR achieving higher Hencky strains than the SER. The lines show the theoretical prediction from the OPP model. (b) The steadystate extensional viscosity values from the FSR and the CSER are compared to the prediction of the Pompom model fitted to the data. 
steady-state stress due to the presence of the overshoot in the model that is not seen experimentally. The reason the OPP model overshoots for these slow strain rates is because the overshoot parameters are kept constant for all modes, but as previously stated we are most interested in the strain-rates measured in the CSER.

In Fig. 9, we show the fit of the OPP model to the HDPE material HDB6. HDB4 is not plotted but is similar. For this material, the FSR is not able to function at the strain-rates used in the CSER, and so for these strain-rates we used the transient data from the SER in fitting the model. As with the LDPE, Dow150R, the transient build up of the extensional viscosity is well captured by the model. As with the Dow 150R material the high strain-rate regime is fitted well by the data, but simultaneously fitting the low strain-rate regime is not possible with a single overshoot parameter $C_{R}$.

In Fig. 10, finite element simulations of Dow150R with the spectrum used in Fig. 8 are compared to the experimental birefringence stress patterns for flow rates giving SP extension-rates of $\dot{\varepsilon}_{C}=0.035 \mathrm{~s}^{-1}, \dot{\varepsilon}_{C}=0.070 \mathrm{~s}^{-1}$, and $\dot{\varepsilon}_{C}=0.174 \mathrm{~s}^{-1}$, correspond to Weissenberg flow numbers of $W_{i}=\dot{\varepsilon}_{C} \bar{\tau}_{b}=15,30$, and 75, respectively (where $\bar{\tau}_{b}$ is the viscosity averaged relaxation time). The simulations show an excellent agreement with the birefringence patterns, predicting not only the number of fringes around the SP but also the shape of the fringe pattern including the correct position of the $\mathrm{W}$-cusps at each flow rate.

We achieve a similar level of agreement for HDB6. The experimental flow rates correspond to strain rates of $\dot{\varepsilon}_{C}=0.70 \mathrm{~s}^{-1}, \dot{\varepsilon}_{C}=1.74 \mathrm{~s}^{-1}$, and $\dot{\varepsilon}_{C}=3.48 \mathrm{~s}^{-1}$ giving Weissenberg numbers of 19.6, 48.7, and 97.4, from top to bottom in Fig. 11. Again, the number of fringes and the shape of the fringe pattern are estimated well. It is also noticeable that the shape of the W-cusps is different between the Dow150R and HDB6. We note that this is not a consequence of orientation Weissenberg number as these are similar. The shape of the cusps for the LDPE Dow150R is highly extended compared to that of HDPE HDB6, as a consequence of the increased level of branching in the LDPE. Also, the W-cusps for Dow150R are also more highly stretched (i.e., the distance from the tip of the double cusps to the centre line dip) compared to HDB6, which is also due to the level of LCB [Auhl et al. (2011)].

The OPP model captures the W-cusp rheology well, predicting both the form of the fringe pattern and the fringe number to within half a fringe for all three Weissenberg
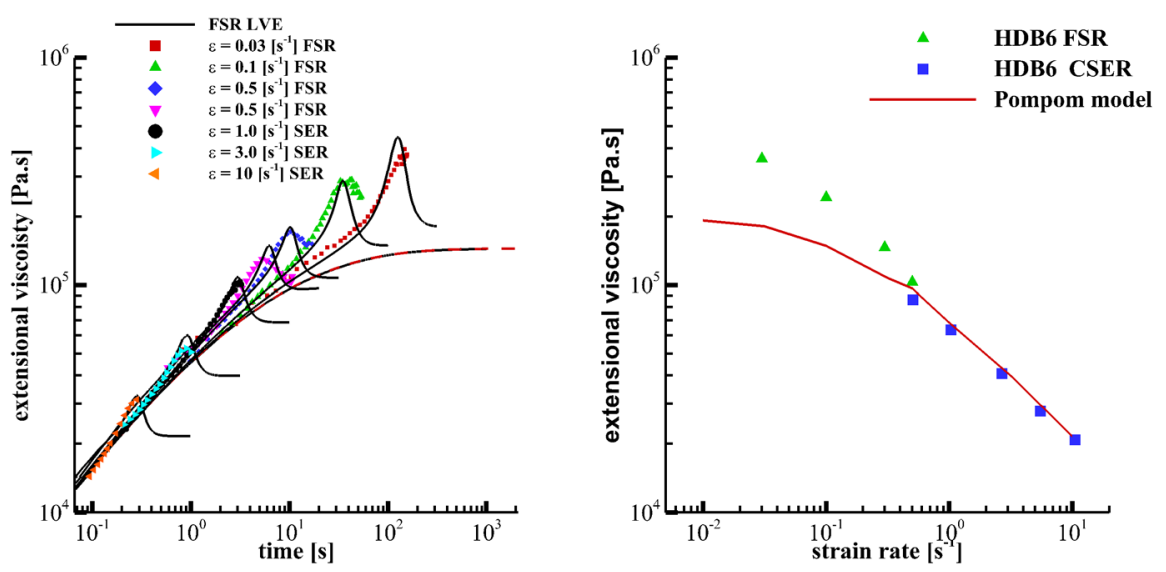

FIG. 9. The transient (a) and steady-state (b) data for HDB6 are plotted along side the OPP model fitted to the data (lines). For the transient fit, the slower strain rates were measured by the FSR and the higher rates by the SER. The steady-state data came from the FSR and the CSER. 


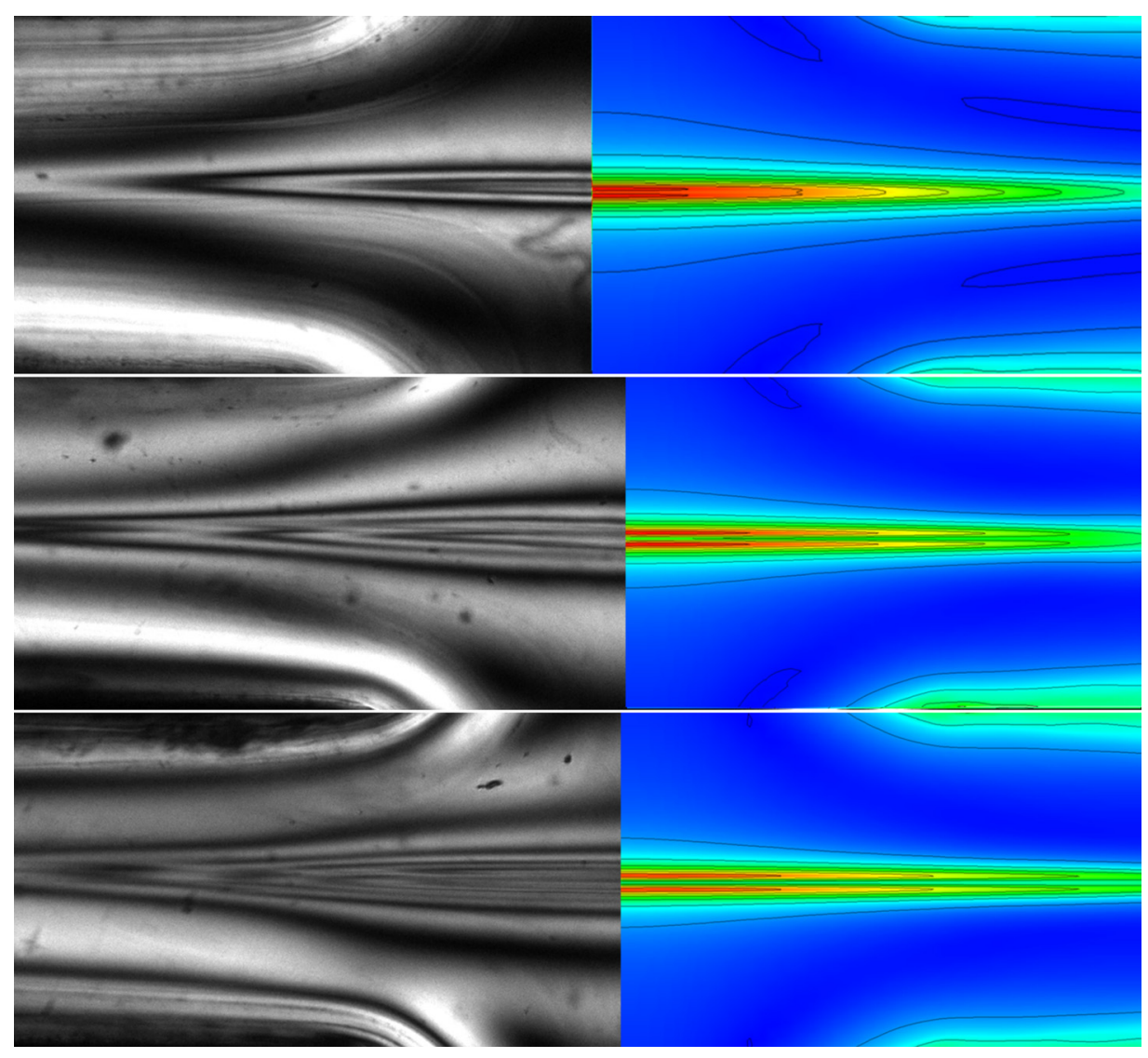

FIG. 10. A comparison of between FIB in cross-slot flow and 2D simulations of the OPP parameterization for LDPE Dow150R. The values of overshoot parameters are $C_{r}=0.8$ and $\alpha=1000$, and the transient extensional rheology is shown in Fig. 8. The black lines in the simulations represent the black contours of the experimental PSD for initial strain rates of $\dot{\varepsilon}_{C}=0.035 \mathrm{~s}^{-1}, \dot{\varepsilon}_{C}=0.070 \mathrm{~s}^{-1}$, and $\dot{\varepsilon}_{C}=0.174 \mathrm{~s}^{-1}$ from top to bottom.

numbers. However, the phenomenology of the OPP model does not account for every detail of the flow. For example, the size of the W-cusps is over predicted near the SP. As with the original Pompom model, the OPP model fails to capture the overall pattern collapse [cf. Hassell and Mackley (2009)] observed for all W-cusping materials. The overall pattern collapse is seen as a decrease in the total number of fringes observed over time. That is, the number of fringes at steady state is usually less than the highest number of fringes observed by a half or one whole fringe. Also the transient development of the pattern is much faster in simulations than in experiments. In experiments, the $\mathrm{W}$-cusps initially occur at a strain of around 5, whereas in transient simulations, not shown, W-cusps first occur at a strain of 2.

The clarity of the steady-state experimental FIB images means that we can determine the PSD as a function of position along the stream line through the SP, which is shown in Fig. 12. These results confirm the visual agreement of the PSD contours. However, the simulations overpredict the stress difference slightly for the two lower Weissenberg numbers and also overpredict the magnitude of PSD overshoot.

Downstream of the SP the stress relaxation of the cusps is not predicted well. Visually, this corresponds to simulations not predicting the appearance of the lower fringe orders away from the SP along the outflow centre line. 


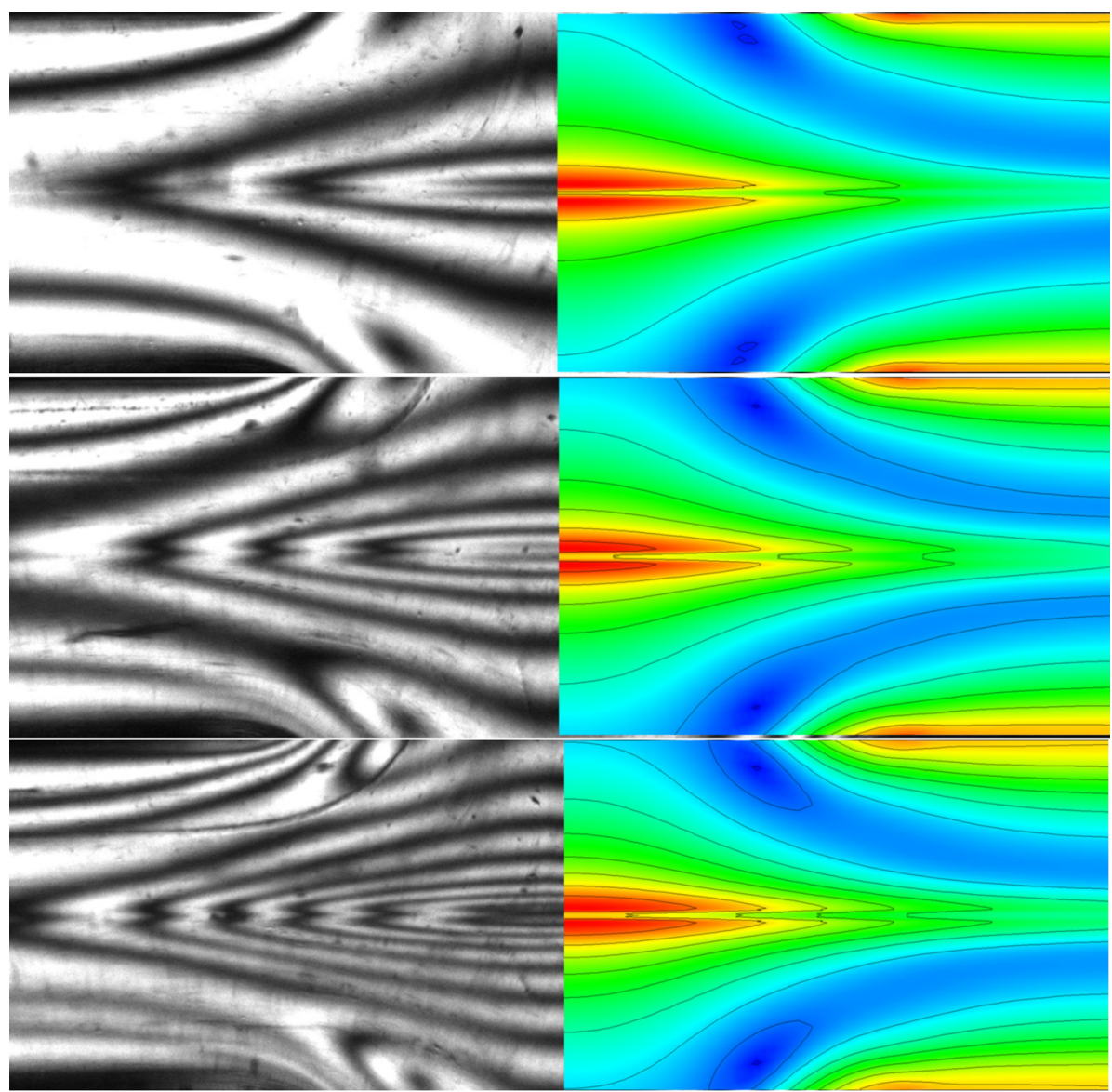

FIG. 11. A comparison of between FIB in cross-slot flow and 2D simulations of the OPP parameterization for HDPE HDB6. The values of overshoot parameters are $C_{r}=2.0$ and $\alpha=1000$, and the transient extensional rheology is shown in Fig. 9. The black lines in the simulations represent the black contours of the experimental PSD for initial strain rates of $\dot{\varepsilon}_{C}=0.70 \mathrm{~s}^{-1}, \dot{\varepsilon}_{C}=1.74 \mathrm{~s}^{-1}$, and $\dot{\varepsilon}_{C}=3.48 \mathrm{~s}^{-1}$ from top to bottom.

\section{CONCLUSIONS}

In this paper, we compared three experimental techniques for measuring the extensional viscosity of LCB polymer melts. The two filament stretching devices (the SER and the FSR) measure startup of uniaxial extensional flow, but with FSR capable of reaching higher Hencky strains due to its feedback control. The CSER measures the steady-state planar extensional viscosity through the stress birefringence at the SP. (The transient viscosity can also be inferred from the stress growth on the incoming stagnation streamline.) All three experimental techniques show excellent agreement for strain-rates at which they can all operate. However, in general the experimental windows of the FSR and the CSER are complimentary to one another with the FSR operating in a low strain-rate regime and the CSER operating in a high strain-rate regime. Hence, we have a robust technique for probing a materials extensional behavior. For the three materials we investigated here, we could accurately characterize the steady-state extensional viscosity. The experiments revealed consistent and striking phenomena unique to strain-hardening melts (seen for all LCB melts); in the FSR, this is manifest as overshoots in the transient stress, which produce W-cusps in the CSER. 


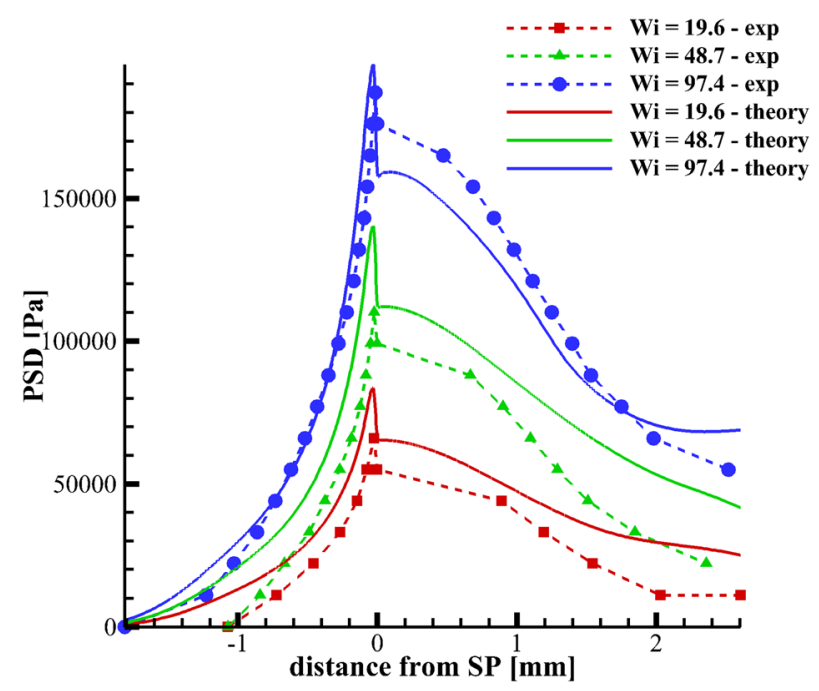

FIG. 12. A comparison between the OPP simulations and the experimentally measured position of the FIB contours of constant PSD for HDB6 at three flow rates.

We have modified the Pompom model to capture this nonmonotonic stress growth in order to fit the full extensional rheology in both transient and steady-state response. Although this modification is not based on molecular physics, it allows us to make a crucial link between the two flow investigated here. By fitting this model to extensional data, we are able to reproduce the birefringence patterns observed in the cross-slot device. From this, we conclude that the spatial $\mathrm{W}$-fringes are the consequence of the temporal stress overshoot in extension of LCB melts. (It should be noted that our extensive investigations with the original Pompom model failed to reproduce the $\mathrm{W}$-fringe pattern.) The current overshoot model, however, fails to capture the relaxation dynamics observed downstream of the SP. A clear future goal would be to derive a constitutive model that is based on molecular detail and captures the full range of characteristics observed in extensional experiments.

\section{ACKNOWLEDGMENTS}

The authors thank the EPSRC Microscale Polymer Processing Research Project (GR/ T11807/01 and GR/T11821/01) and the European Union Marie Curie Initial Training Network DYNACOP (214627) for their financial support.

\section{References}

Abedijaberi, A., J. Soulages, M. Kröger, and B. Khomami, "Flow of branched polymer melts in a lubricated cross-slot channel: A combined computational and experimental study," Rheol. Acta 48, 97-108 (2009).

Aho, J., V. H. Rolon-Garrido, S. Syrjala, and M. H. Wagner, "Extensional viscosity in uniaxial extension and contraction flow-Comparison of experimental methods and application of the molecular stress function model," J. Non-Newtonian Fluid Mech. 165(5-6), 212-218 (2010a).

Aho, J., V. H. Rolon-Garrido, S. Syrjala, and M. H. Wagner, "Measurement technique and data analysis of extensional viscosity for polymer melts by Sentmanat extensional rheometer (SER)," Rheol. Acta 49(4), 359-370 (2010b).

Auhl, D., D. M. Hoyle, D. Hassell, T. D. Lord, O. G. Harlen, M. R. Mackley, and T. C. B. McLeish, "Cross-slot extensional rheometry and the steady-state extensional response of long chain branched polymer melts," J. Rheol. 55(4), 875-900 (2011). 
Bach, A., H. K. Rasmussen, and O. Hassager, "Extensional viscosity for polymer melts measured in the filament stretching rheometer,” J. Rheol. 47(2), 429-441 (2003).

Bent, J., L. R. Hutchings, R. W. Richards, T. Gough, R. Spares, P. D. Coates, I. Grillo, O. G. Harlen, D. J. Read, R. S. Graham, A. E. Likhtman, D. J. Groves T. M. Nicholson, and T. C. B. McLeish, "Neutron-mapping polymer flow: Scattering, flow visualisation and molecular theory," Science 301, 1691-1695 (2003).

Blackwell, R. J., O. G. Harlen, and T. C. B. McLeish, "Molecular drag-strain coupling in branched polymer melts," J. Rheol. 44(1), 121-136 (2000).

Bogaerds, A. C. B., W. M. H. Verbeeten, G. W. M. Peters, and F. P. T. Baaijens, "3D viscoelastic analysis of a polymer solution in a complex flow," Comput. Methods Appl. Mech. Eng. 180, 413-430 (1999).

Boukellal, G., A. Durin, R. Valette, and J. Agassant, "Evaluation of a tube based constitutive equation using conventional and planar elongation flow optical rheometers," Rheol. Acta 50, 547-557 (2011).

Clemeur, N., R. P. G. Rutgers, and B. Debbaut, "On the evaluation of some differential formulations for the pompom constitutive model,” Rheol. Acta 42, 217-231 (2003).

Clemeur, N., R. P. G. Rutgers, and B. Debbaut, "Numerical evaluation of three dimensional effects in planar flow birefringence," J. Non-Newtonian Fluid Mech. 123, 105-120 (2004).

Cogswell, F. N., "Converging flow of polymer melts in extrusion dies," Polym. Eng. Sci. 12(1), 64-73 (1972).

Collis, M. W., and M. R. Mackley, "The melt processing of monodisperse and polydisperse polystyrene melts within a slit entry and exit flow," J. Non-Newtonian Fluid Mech. 128(1), 29-41 (2005).

Coventry, K. D., and M. R. Mackley, "Cross-slot extensional flow birefringence observations of polymer melts using a multi-pass rheometer,” J. Rheol. 52(2), 401-415 (2008).

Crowley, D. G., F. C. Frank, M. R. Mackley, and R. G. Stephenson, "Localised flow birefringence of polyethylene oxide solutions in a four roll mill,” J. Polym. Sci., Part B: Polym. Phys. 14(6), 1111-1119 (1976).

Das, C., N. J. Inkson, D. J. Read, and K. Kelmanson, "Computational linear rheology of general branch-onbranch polymers,” J. Rhol. 50(2), 207-234 (2006).

Dealy, J. M., “Do polymeric liquids exhibit strain hardening?,” J. Rheol. 34(7), 1133-1147 (1990).

Frank, F. C., and M. R. Mackley, "Localized flow birefringence of polyethylene oxide solutions in a two roll mill,” J. Polym. Sci., Part A-2 14, 1121-1131 (1976).

Hassell, D. G., and M. R. Mackley, "Localised flow induced crystallisation of a polyethylene melt," Rheol. Acta 47(4), 435-446 (2008).

Hassell, D. G., and M. R. Mackley, "An experimental evaluation of the behaviour of mono and polydisperse polystyrenes in cross-slot flow," Rheol. Acta 48, 543-550 (2009).

Hassell, D. G., D. Hoyle, D. Auhl, O. Harlen, M. R. Mackley, and T. C. B. McLeish, "Effect of branching in cross-slot flow: The formation of W cusps," Rheol. Acta 48, 551-561 (2009).

Hassell, D. G., D. Auhl, T. C. B. McLeish, O. G. Harlen, and M. R. Mackley, "The effect of viscoelasticity on stress fields within polyethylene melt flow for a cross-slot and contraction-expansion slit geometry," Rheol. Acta 47, 821-834 (2008).

Hoyle, D. M., “Constitutive modelling of branched polymer melts in non-linear response," Ph.D. thesis, University of Leeds, Leeds, 2011.

Inkson, N. J., T. C. B. McLeish, O. G. Harlen, and D. J. Groves, "Predicting low density polyethylene melt rheology in elongational and shear flows with pompom constitutive equations," J. Rheol. 43(4), 873-896 (1999).

Janeschitz-Kriegl, H., Polymer Melt Rheology and Flow Birefringence (Springer, Berlin, 1983).

Kolte, M. I., H. K. Rasmussen, and O. Hassager, "Transient filament stretching rheometer. 2. Numerical simulation," Rheol. Acta 36, 285-302 (1997).

Kotaka, T., A. Kojima, and M. Okamoto, "Elongational flow opto-rheometry for polymer melts. 1. Construction of an elongational flow opto-rheometer and some preliminary results," Rheol. Acta 36, 646-656 (1997).

Koyama, K., and O. Ishizuka, "Birefringence of polyethylene melt in transient elongational flow at constant strain rate,” J. Polym. Sci., Part B: Polym. Phys. 27, 297-306 (1989).

Laun, H. M., and H. Münstedt, "Comparison of the elongational behaviour of a polyethylene melt at constant stress and constant strain rate," Rheol. Acta 15(10), 517-524 (1976).

Laun, H. M., and H. Münstedt, "Elongational behaviour of a low density polyethylene melt," Rheol. Acta 17, 415-425 (1978). 
Laun, H. M., and H. Schuch, “Transient elongational viscosities and drawability of polymer melts," J. Rheol. 33(1), 119-175 (1989).

Lord, T. D., L. Scelsi, D. G. Hassell, M. R. Mackley, J. Embery, D. Auhl, O. G. Harlen, R. Tenchev, P. K. Jimack, and M. A. Walkley, "The matching of 3d rolie-poly viscoelastic numerical simulations with experimental polymer melt flow within a slit and a cross-slot geometry," J. Rheol. 54, 355-373 (2010).

Mackley, M. R., R. T. J. Marshall, and J. B. A. F. Smeulders, “The multipass rheometer,” J. Rheol. 39(6), 12931309 (1995).

Macosko, C. W., Rheology, Principles, Measurements and Applications (Wiley-VCH, New York, 1994).

Macosko, C. W., M. A. Ocansey, and H. H. Winter, "Studies with lubricated planar stagnation dies," J. Rheol. 24(6), 958-958 (1980).

McKinley, G. H., and T. Sridhar, "Filament-stretching rheometry of complex fluids," Annu. Rev. Fluid Mech. 34, 375-415 (2002).

McLeish, T. C. B., "Tube theory of entangled polymers," Adv. Phys. 51, 1379-1527 (2002).

McLeish, T. C. B., and R. G. Larson, "Molecular constitutive equations for a class of branched polymers: The pompom polymer," J. Rheol. 42(1), 81-110 (1998).

Meissner, J., "Dehnungsverhalten von Polyäthylen-Schmelzen,” Rheol. Acta 10, 230-242 (1971).

Meissner, J., "Experimental aspects in polymer melt elongational rheometry," Chem. Eng. Commun. 33, 159-180 (1985).

Meissner, J., and J. Hostettler, "A new elongational rheometer for polymer melts and other highly viscoelastic liquids," Rheol. Acta 33, 1-21 (1994).

Meissner, J., S. E. Stephenson, A. Demarmels, and P. Portmann, "Multiaxial elongational flows of polymer melts—classification and experimental realization,” J. Non-Newtonian Fluid Mech. 11, 221-237 (1982).

Meissner, J., T. Raible, and S. E. Stephenson, "Rotary clamp in uniaxial and biaxial extensional rheometry of polymer melts," J. Rheol. 25, 1-28 (1981).

Minoshima, W., and J. L. White, "A comparative experimental study of the isothermal shear and elongational rheological properties of low density, high density and linear low density polyethylenes," J. Non-Newtonian Fluid Mech. 19, 251-274 (1986a).

Minoshima, W., and J. L. White, "Instability phenomena in tubular film and melt spinning of rheologically characterised high density, low density and linear low density polyethylenes," J. Non-Newtonian Fluid Mech. 19, 275-302 (1986b).

Münstedt, H., "New universal extensional rheometer for polymer melts. Measurements on polystyrene," J. Rheol. 23(4), 421-436 (1979).

Münstedt, H., and D. Auhl, "Rheological measuring techniques and their relevance for the molecular characterization of polymers," J. Non-Newtonian Fluid Mech. 128, 62-69 (2005).

Münstedt, H., and H. M. Laun, "Elongational properties and molecular structure of polyethylene melts," Rheol. Acta 20, 211-221 (1981).

Nielsen, J. K., H. K. Rasmussen, M. Denberg, K. Almdal, and O. Hassager, "Nonlinear branch-point dynamics of multiarm polystyrene," Macromolecules 39(25), 8844-8853 (2006).

Öttinger, H. C., "Thermodynamic admissibility of the pompom model for branched polymers," Rheol. Acta 40, 317-321 (2001).

Raible, T., A. Demarmels, and J. Meissner, "Stress and recovery maxima in LDPE melt elongation," Polym. Bull. 1(6), 397-402 (1979).

Rasmussen, H. K., A. G. Bejenariu, O. Hassager, and D. Auhl, "Experimental evaluation of the pure configurational stress assumption in the flow dynamics of entangled polymer melts," J. Rheol. 54, 1325-1336 (2010).

Rasmussen, H. K., J. K. Nielson, A. Bach, and O. Hassager, "Viscosity overshoot in the start-up of uniaxial elongation of low density polyethylene melts," J. Rheol. 49(2), 369-381 (2005).

Rolon-Garrido, V. H., and M. H. Wagner, "The damping function in rheology," Rheol. Acta 48(3), 245-284 (2009).

Schoonen, J. F. M., F. H. M. Swartjes, G. W. M. Peters, F. P. T. Baaijens, and H. E. H. Meijer, “A 3D numerical/experimental study on a stagnation flow of a polyisobutylene solution," J. Non-Newtonian Fluid Mech. 79(2-3), 529-561 (1998). 
Scrivener, O., C. Bernera, R. Cressely, R. Hocquart, R. Sellin, and N. S. Vlachos, "Dynamical behaviour of drag-reducing polymer solutions,” J. Non-Newtonian Fluid Mech. 5, 475-495 (1979).

Sentmanat, M. L., "Miniature universal testing platform: From extensional melt rheology to solid-state deformation behavior," Rheol. Acta 43(6), 657-669 (2004).

Soulages, J., T. Schweizer, D. C. Venerus, J. Hostettler, F. Mettler, M. Kröger, and H. C. Öttinger, "Lubricated optical rheometer for the study of two-dimensional complex flows of polymer melts," J. Non-Newtonian Fluid Mech. 150, 43-55 (2008).

Spiegelberg, S. H., D. C. Ables, and G. H. McKinley, "The role of end-effects on measurements of extensional viscosity in filament stretching rheometers," J. Non-Newtonian Fluid Mech. 64, 229-267 (1996).

Sridhar, T., V. Tirtaatmadja, D. A. Nguyan, and R. K. Gupta, "Measurement of extensional viscosity of polymer solutions,” J. Non-Newtonian Fluid Mech. 40(3), 271-280 (1991).

Szabo, P., "Transient filament stretching rheometer. 1. Force balance analysis," Rheol. Acta 36, 277-284 (1997).

Tenchev, R., O. G. Harlen, P. K. Jimack, and M. A. Walkley, "Finite element modelling of two- and threedimensional viscoelastic polymer flows," in Trends in Engineering Computational Technology, edited by M. Papadrakakis, and B. H. V. Topping (Saxe-Coburg, Edinburgh, UK, 2008), pp. 81-101.

Verbeeten, W. M. H., G. W. M. Peters, and F. P. T. Baaijens, "Differential constitutive equations for polymer melts: The extended pompom model," J. Rheol. 45(4), 823-843 (2001).

Wagner, M. H., and V. H. Rolon-Garrido, "Verification of branch point withdrawal in elongational flow of pompom polystyrene melt,” J. Rheol. 52(5), 1049-1068 (2008).

Winter, H. H., C. W. Macosko, and K. E. Bennett, "Orthogonal stagnation flow, a framework for steady extensional flow experiments," Rheol. Acta 18, 323-334 (1979). 